\title{
Effectiveness of Neurodynamic Mobilization versus Conventional Therapy on Spasticity Reduction and Upper Limb Function in Tetraplegic Patients
}

\author{
Akanksha Saxena $^{1}$, Stuti Sehgal ${ }^{2}$, Mandeep Kumar Jangra ${ }^{3}$ \\ ${ }^{1}$ Department of Physiotherapy, Maharishi Markandeshwar University, Mullana, India \\ ${ }^{2}$ Division of Neurology, Department of Physiotherapy, Indian Spinal Injuries Center-Institute of Rehabilitation Sciences, New Delhi, India \\ ${ }^{3}$ Division of Cardiothoracic \& Pulmonary Disorders, Department of Physiotherapy, Maharishi Markandeshwar University, Mullana, India
}

Study Design: The study employed a pre- and post-test experimental design.

Purpose: This study was designed to assess the effect of neurodynamic mobilization of the median nerve on upper limb spasticity in tetraplegic patients.

Overview of Literature: Spasticity is a common and potentially disabling and bothersome complication in patients with spinal cord lesion; this disorder can negatively influence the quality of life by restricting the patient's ability to perform activities of daily living. Neural mobilization is currently used for reducing the spasticity in individuals with neurological disorders.

Methods: Twenty subjects with traumatic spinal cord injury (level C5-C8) and upper limb spasticity in the finger and wrist flexors were enrolled. They were randomly allocated to two different groups using a computer-generated randomization schedule: group I comprised the neurodynamic mobilization group ( $n=11)$ and group II was the conventional therapy group ( $n=9)$; the subjects were administered therapy for 5 days every week for a period of 4 weeks. Upper limb spasticity was assessed using the Modified Ashworth Scale for wrist and finger flexors; F-wave amplitude, latency, and F-wave/M-wave amplitude ratio (F/M ratio) were examined using the F-wave scores of the median nerve; and upper limb function was determined using the Capabilities of Upper Extremity (CUE) Questionnaire.

Results: After 4 weeks of intervention, between-group comparisons showed a significant difference in the pre-intervention and postintervention scores on the Modified Ashworth Scale score for wrist flexors (-1.64 \pm 0.67$)$, Modified Ashworth Scale score for finger

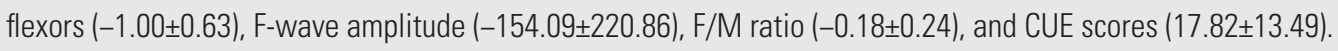

Conclusions: These results suggest that neurodynamic mobilization of the median nerve may be effective for upper limb spasticity control and upper limb functional improvement in tetraplegic patients.

Keywords: Upper limb; Spasticity; F-wave; Spinal cord injuries; Neurodynamic mobilization

Received Apr 3, 2020; Revised May 27, 2020; Accepted May 28, 2020

Corresponding author: Akanksha Saxena

Maharishi Markandeshwar University of Health Sciences, Flat no. 61, D-block, Mullana, Ambala (Haryana)-133203, India

Tel: +91-7906989678, Fax: +91-9958729161, E-mail: akankshasaxena623@gmail.com; akankshasaxena623@mmumullana.org 


\section{Introduction}

Spinal cord injuries (SCIs) interrupt the axonal pathways and segmental spinal cord connections at and below the level of the injury, leading to severe motor, sensory, and autonomic dysfunction [1]. SCI patients experience many major complications, depending on the level and severity of the lesion, such as paralysis, spasticity, sensory loss, intractable pain, pressure sores, and urinary and other infections that lower their health-related quality of life [2]. Spasticity is a common complication followed by SCI that potentially limits functional independence [3].

Spasticity is reported to be one of the most difficult health complications after SCI [4]. Moreover, 65\%-78\% of subjects with chronic SCI ( $\geq 1$ year post injury) show symptoms of spasticity [5]. The most common anatomical region of the injury was the cervical spine (43.9\%-61.5\%) causing tetraplegia [5]. Moreover, $60 \%$ of those with a cervical injury had spasticity in their upper extremities. Spasticity can negatively affect the quality of life by restricting activities of daily living (ADLs); inhibiting efficient walking and self-care; causing pain and fatigue; disturbing sleep; and hampering safety, leading to the development of contractures, pressure ulcers, infections, and negative self-image, thus complicating the role of the caretaker and impeding rehabilitation efforts [5]. The major concern in cervical SCI patients is upper limb spasticity because it can cause barriers in restoring function by hampering the ADLs that, in turn, adversely affect the level of independence [6]. The management of spasticity is desirable for the control of passive problems, like reducing pain, facilitating splint wearing, easing positioning and hygiene, and preventing contractures, or functional problems, including the individual's decreased ability to perform important motor functions [5].

Systemic pharmacological treatment of spasticity is frequently prescribed for patients with SCI, including baclofen, tizanidine, and gabapentin; these may have possible adverse effects, such as dizziness, nausea, withdrawal seizures, hallucinations, and ataxia; no single medication has shown a positive effect in all patients [4]. Rehabilitation is considered necessary in the management of spasticity as a long-term regimen along with surgical and pharmacological interventions. The goal is to diminish spasticity and allow voluntary movements and/or to improve the ability to independently perform ADLs, such as transfers, dressing, and toileting [4].
Neural mobilization is a currently used technique that aims to reduce the spasticity in patients with neurological disorders. Neural mobilization refers to a group of techniques that aim to place the neuraxis in tension and stretch it with appropriate mobilization through certain postures, along with the application of slow, rhythmic movements of the joints intended to reach the peripheral nerves and the spinal cord [7]. These techniques are developed gradually, using the diagnostic tests proposed by Elvey and Hall [8] to assess adverse neural tension.

The main purpose of neural mobilization is to restore the dynamic balance between the movement of neural tissue and mechanical connections of the surroundings, to promote optimal physiological function, and to restore the normal mechanical and physiological state of motion and posture [7,9]. These movements allow the maintenance, elasticity, and extensibility of the nervous system, thus enhancing muscle maintenance and extensibility [10].

F-wave is a compound action potential elicited by the supra-maximal antidromic stimulation of a motor nerve after the direct muscle response. They are useful in the assessment of proximal conduction slowing, as evident in spasticity [11]. Rosche et al. [12] showed that F-wave amplitude and $\mathrm{F}$-wave/M-wave amplitude ratio (F/M ratio) can be used to document spasticity.

This study was designed to test the hypothesis that neurodynamic mobilization is effective in reducing spasticity of the upper limbs and improving hand function in tetraplegic patients.

\section{Materials and Methods}

The study employed a pre- and post-test experimental design.

\section{Participants}

We used convenience sampling to enroll 22 tetraplegic subjects with traumatic SCI in our study as per the inclusion and exclusion criteria. The subjects were recruited from the rehabilitation department, Indian Spinal Injuries Centre, Vasant Kunj, New Delhi after they provided written consent for study participation. Twenty subjects completed the intervention successfully, and two subjects dropped out (one dropped out after 2 days of treatment and the other after 4 days due to their inability to complete the study). One hand of each subject was given the 
intervention based on the inclusion/exclusion criteria. In patients for whom both hands met the criteria, the hand with greater spasticity was chosen for the study.

\section{Inclusion criteria}

Subjects with traumatic SCI [13]; with American Spinal Injury Association (ASIA) impairment grade A, B, C, and $\mathrm{D}$ [4]; those who could lie in the supine position [2]; those who were oriented and alert; those aged 18-65 years [6]; those with non-progressive SCI and residual neurological deficits [14]; and those with complete or incomplete SCI were enrolled.

\section{Exclusion criteria}

Subjects with a score $>3$ on the Modified Ashworth Scale [2]; those who did not provide signed informed consent [2]; those with symptomatic zygapophyseal joints of the cervical spine [15]; those experiencing dizziness [15]; those with pathologies that affected the nervous system, such as diabetes, multiple sclerosis, and Guillian-Barre syndrome; those with a recent history of any surgery [15]; and subjects with Cauda Equina lesions [15] were excluded.

\section{Procedure}

A detailed explanation of the study and a detailed patient information sheet was given to all the subjects. Written informed consent was obtained from all the study subjects. The study protocol was reviewed and approved by the Institutional Ethical Committee (ISIC/IIRS/RP/2015/068).

Demographic data, neurological details, and baseline characteristics of the subjects were collected using the evaluation proforma, ASIA form for SCI to determine the neurological level of the SCI and International Spinal Cord Injury Core Data Set form. F-wave for stimulating median nerve (abductor pollicis brevis muscle) was performed by an experienced neuro-electrophysiological technician working at the neuro-electrophysiological department of Indian Spinal Injuries Centre. Subjects were then randomly and equally divided into the following groups using a fixed random allocation method: group I comprised the neural mobilization group and group II was the conventional group. Before the intervention, subjects from both the groups were assessed by a blinded assessor, and scores were obtained for the following out- come measures: (1) scores for spasticity of the upper limb of choice (Modified Ashworth Scale score for wrist flexors and finger flexors); (2) score on Capabilities of Upper Extremity (CUE) Questionnaire; and (3) F-wave parameters (F-wave amplitude; minimum, maximum, and mean latency and; F/M ratio).

Group I underwent neurodynamic mobilization of the median nerve. Mobilization was performed for all the subjects for 12 minutes during each session; sessions were conducted 5 times each week for 4 weeks (total 20 sessions) from the time of study initiation. Each subject was made to lie in the supine on the plinth with the scapula free of the bed. With the maintenance of shoulder girdle depression, the glenohumeral joint was extended, abducted, and laterally rotated; the elbow was extended; the forearm was supinated; and the wrist, fingers, and thumb were extended. After holding this position, neural mobi-

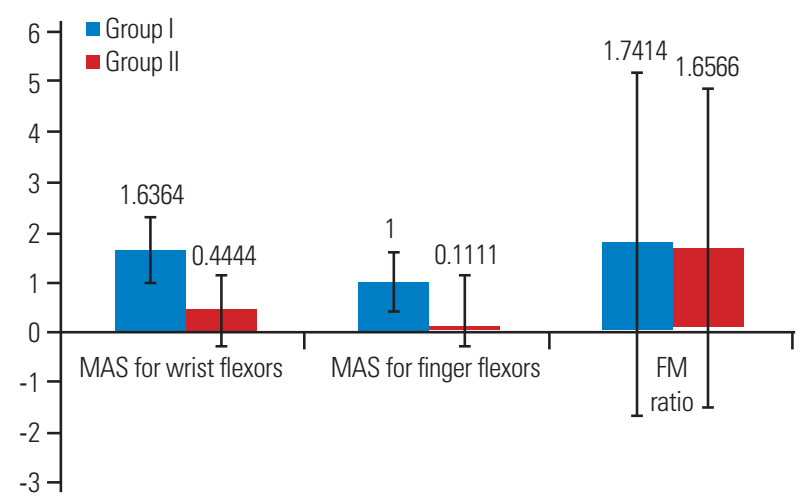

Fig. 1. Mean difference of Modified Ashworth Scale score (MAS) for wrist \& finger flexors and F-wave/M-wave amplitude (F/M) ratio scores.

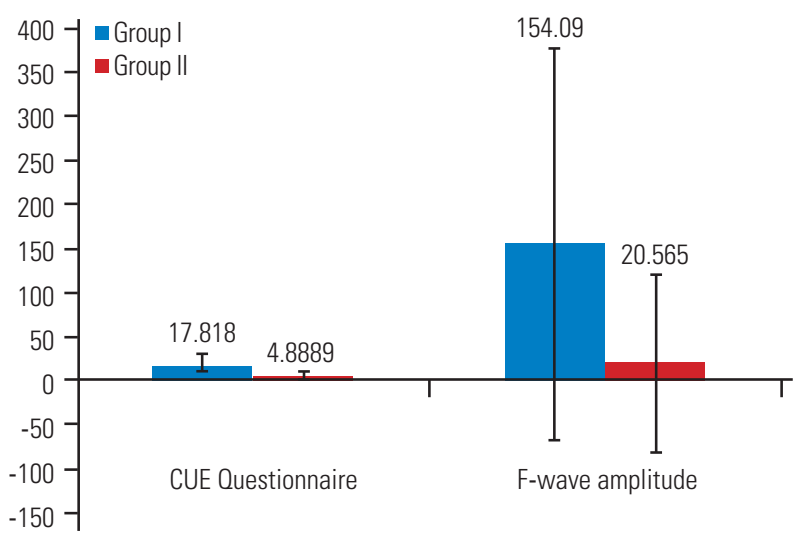

Fig. 2. Mean difference of F-wave amplitude \& Capabilities of the Upper Extremity (CUE) Questionnaire scores. Mean difference scores are the mean of the difference between post- and pre-intervention scores. 
lization was performed with slow, rhythmic oscillations of wrist flexion and extension. Twenty oscillations were performed each minute for 3 minutes; the process was performed thrice during the same session, with a 1-minute interval between consecutive attempts [13].

Group II received conventional therapy. Stretching was performed for all subjects for 15 minutes in every session; we conducted sessions 5 times every week for a period of 4 weeks (total 20 sessions) from the time of study initiation. Each subject was made to lie in the supine position on the plinth with the scapula free of the bed. The subject's shoulder was in abduction, elbow in extension, wrist in dorsiflexion, and fingers in extension. After holding this position, stretch was maintained for 1 minute and repeated 9 times during every session [13]. The assessor was blinded to the type of intervention given to the subjects. All the interventions were provided by the same therapist. Post-intervention scores were obtained after 28 days of study initiation, that is, on the 29th day of the study for all the outcome measures in the same manner as that during the pre-intervention assessment.

All the data were analyzed using IBM SPSS ver. 21.0 (IBM Corp., Armonk, NY, USA). Wilcoxon signedrank test was used for within-group analyses, and MannWhitney $U$-test was used for between-group analyses. Statistical significance was set at $p \leq 0.05$, and the value of the confidence interval was set at $95 \%$.

\section{Results}

A total of 20 subjects participated in the study. The demo-

graphic characteristics of the study population are summarized in (Table 1).

\section{Within group analysis}

Our results showed significant differences between the pre-intervention and post-intervention scores of the Modified Ashworth Scale for wrist and finger flexors, F-wave amplitude, and scores of the CUE in group I and scores of

Table 1. Demographic details of groups I and II

\begin{tabular}{|c|c|c|}
\hline Characteristic & Group I (n=11) & Group II $(n=9)$ \\
\hline Age (yr) & $28.64 \pm 6.96$ & $35.22 \pm 11.49$ \\
\hline Time since injury (mo) & $21.14 \pm 17.39$ & $9.89 \pm 5.75$ \\
\hline \multicolumn{3}{|l|}{ Gender } \\
\hline Males & 10 & 9 \\
\hline Females & 1 & 0 \\
\hline \multicolumn{3}{|l|}{ Neurological level } \\
\hline $\mathrm{C} 5$ & 6 & 5 \\
\hline C6 & 5 & 3 \\
\hline C7 & - & 1 \\
\hline \multicolumn{3}{|l|}{ AIS } \\
\hline AIS A & 3 & 4 \\
\hline AIS B & 3 & 4 \\
\hline AIS C & - & 1 \\
\hline AIS D & 3 & - \\
\hline
\end{tabular}

Table 2. Comparison of difference between pre- and post-intervention scores between group I and group II

\begin{tabular}{|c|c|c|c|c|}
\hline \multirow{2}{*}{ Variable } & \multicolumn{2}{|c|}{ Difference between pre- \& post-intervention scores } & \multirow{2}{*}{$Z$-value } & \multirow{2}{*}{$p$-value (1-tailed) } \\
\hline & Group I ( $n=11)$ & Group II (n=9) & & \\
\hline MAS for wrist flexors & $-1.6364 \pm 0.67420$ & $-0.4444 \pm 0.72648$ & -3.035 & $0.003^{*}$ \\
\hline MAS for finger flexors & $-1.0000 \pm 0.63246$ & $-0.1111 \pm 0.33333$ & -3.035 & $0.004^{*}$ \\
\hline FL minimum & $0.1409 \pm 1.59935$ & $-0.3278 \pm 3.30370$ & -0.114 & 0.941 \\
\hline FL maximum & $-0.3864 \pm 2.22093$ & $-0.8778 \pm 3.19874$ & -0.342 & 0.766 \\
\hline FL mean & $-0.0964 \pm 1.72186$ & $-0.6033 \pm 3.36348$ & -0.038 & 1.00 \\
\hline F-reflex amplitude & $-154.09 \pm 220.85897$ & $20.565 \pm 99.46174$ & -2.566 & $0.010^{*}$ \\
\hline F-wave/M-wave amplitude ratio & $1.7414 \pm 3.43270$ & $-1.6566 \pm 3.18460$ & -1.026 & 0.331 \\
\hline CUE Questionnaire & $17.818 \pm 13.48939$ & $4.8889 \pm 4.37163$ & -2.587 & $0.007^{*}$ \\
\hline
\end{tabular}

Values are presented as mean \pm standard deviation.

MAS, Modified Ashworth Scale score; FL, F-reflex latency; CUE, Capabilities of Upper Extremity.

* $p<0.05$ (statistically significant). 
the CUE scores in group II.

\section{Between-group analysis}

There were significant differences between the pre-intervention and post-intervention scores of the Modified Ashworth Scale for wrist and finger flexors, F-wave amplitude, and score of the CUE in group I (Table 2, Figs. 1, 2).

\section{Discussion}

The present findings were consistent with a previous report by Solorzano [16] as per which neurodynamic mobilization of the median nerve is effective in decreasing the spasticity in upper limb muscles of SCI patients. The neurodynamic mobilization treatment administered in this study was adapted from the study by Solorzano [16] that used the findings of two studies conducted by Godoi et al. [17] and Castilho et al. [7]. Castilho et al. [7] found a significant decrease in the electromyography activity of the biceps brachii immediately after the intervention of neural mobilization of the median nerve in stroke patients. Solorzano [16] found a significant reduction in the Modified Ashworth Scale score, improvement in the joint range of motion, Action Research Arm Test and in Functional Independence Measure after 3 weeks of neural mobilization in a case study on a quadriplegia patient. Consistent with the results of both studies, clinically meaningful improvements were observed in the F-wave amplitude, F/M ratio, Modified Ashworth Scale score, and CUE score. Our results were in agreement with those reported by Solorzano [16] and suggested that a longer intervention program may be more effective.

As Butler and Jones [18] stated "central and peripheral nervous systems are considered one system upheld by three dimensions: mechanical, electrical, and chemical continuity"; it is assumed that after an injury to the nervous system, tension increases and negatively affects the patient's mobility and functional ability. If the neural tension increases unusually, the muscle tone is altered and is markedly increased in the distal segments of the extremities [16].

Marinzeck [19] stated that the use of upper limb neurodynamic test 1 improves retrograde axoplasmic flow, which is abnormal in spasticity, thereby alleviating nerve tension, reducing restrictions and adhesions of the nerve to the surrounding tissues, thus improving the conditions for the enhancement of activities and muscle nutrition. The hypothetical benefits of neurodynamic mobilization include facilitating nerve sliding, reducing neural adhesion, dispersing harmful liquids, increasing nerve vascularization, and improving axoplasmic flow [19]. All these physiological functions of neural tissues are compromised in patients with spasticity; therefore, we can conclude that neurodynamic mobilization reduces spasticity. This is consistent with the results of our study wherein neurodynamic mobilization reduced spasticity, as shown by the reductions in the F-wave amplitude, F/M ratio, and Modified Ashworth Scale scores.

The upper limb function improved in both, the neural mobilization group and the conventional therapy group. However, the upper limb function scores, including the CUE score, showed more significant improvements in the neural mobilization group than in the conventional group. Cowell and Phillips [20] in 2002 reported that the nerve mobilization technique improves the nervous system structure and muscle flexibility [21]. Similarly, we found that improvement in median nerve flexibility might contribute to improved upper limb function. The improvement in the CUE score in our study was similar to that reported by Cha et al. [22] in 2014. In his study on 22 stroke patients, he found that sciatic nerve mobilization improved lower limb function after 4 weeks of sciatic nerve mobilization intervention [22].

The conventional therapy group showed no significant differences in spasticity. Neurodynamic mobilization is an effective method to resolve the issue of spasticity because it is less time consuming unlike stretching and weight bearing, is cost effective, and does not need any equipment or machine.

Although this study showed that neural mobilization helps reduce spasticity, the study also has certain limitations. First, we employed a relatively small sample size. Second, only trauma cases of SCI were included. Therefore, our results cannot be generalized to non-trauma cases. Finally, we did not perform long-term follow up.

\section{Conclusions}

Neurodynamic mobilization of the median nerve is more effective than conventional therapy in reducing upper limb spasticity in patients with traumatic SCI, as measured using F-wave and the Modified Ashworth Scale. The upper limb function, as measured using the CUE score, 
was improved in both the groups.

\section{Conflict of Interest}

No potential conflict of interest relevant to this article was reported.

\section{References}

1. Lynskey JV, Belanger A, Jung R. Activity-dependent plasticity in spinal cord injury. J Rehabil Res Dev 2008;45:229-40.

2. Rossignol S, Schwab M, Schwartz M, Fehlings MG. Spinal cord injury: time to move? J Neurosci 2007;27:11782-92.

3. Biering-Sorensen F, Nielsen JB, Klinge K. Spasticityassessment: a review. Spinal Cord 2006;44:708-22.

4. Westerkam D, Saunders LL, Krause JS. Association of spasticity and life satisfaction after spinal cord injury. Spinal Cord 2011;49:990-4.

5. Adams MM, Hicks AL. Spasticity after spinal cord injury. Spinal Cord 2005;43:577-86.

6. Bryden AM, Peljovich AE, Hoyen HA, Nemunaitis G, Kilgore KL, Keith MW. Surgical restoration of arm and hand function in people with tetraplegia. Top Spinal Cord Inj Rehabil 2012;18:43-9.

7. Castilho J, Ferreira LAB, Pereira WM, et al. Analysis of electromyographic activity in spastic biceps brachii muscle following neural mobilization. J Bodyw Mov Ther 2012;16:364-8.

8. Elvey RL, Hall TM. Neural tissue evaluation and treatment. In: Donatelli RA, editor. Physical therapy of the shoulder. 5th ed. St. Louis (MO): Elsevier Churchill Livingstone; 2011. p. 187-203.

9. Sambyal S, Kumar S. Comparison between nerve mobilization and conventional physiotherapy in patients with cervical radiculopathy. Int J Innov Res Dev 2013;2:442-5.

10. Ellis RF, Hing WA. Neural mobilization: a systematic review of randomized controlled trials with an analysis of therapeutic efficacy. J Man Manip Ther 2008;16:8-22.

11. Eisen A, Fisher M. The F wave: the International Federation of Clinical Neurophysiology. Electroencephalogr Clin Neurophysiol Suppl 1999;52:255-7.
12. Rosche J, Rub K, Niemann-Delius B, Mauch E, Kornhuber HH. Effects of physiotherapy on F-waveamplitudes in spasticity. Electromyogr Clin Neurophysiol 1996;36:509-11.

13. Singh A, Tetreault L, Kalsi-Ryan S, Nouri A, Fehlings MG. Global prevalence and incidence of traumatic spinal cord injury. Clin Epidemiol 2014;6:309-31.

14. Skold C, Levi R, Seiger A. Spasticity after traumatic spinal cord injury: nature, severity, and location. Arch Phys Med Rehabil 1999;80:1548-57.

15. Zubieta C. Influence of rhythmical mobilization of brachial plexus using the neurodynamic test ULNT1 with variation of the components on the muscle tone of neurological patients with upper limb hypertonia and difficulty to open their hand derived there from [dissertation]. Torrelavega: Gimbernat School of Physiotherapy; 2013.

16. Solorzano CB. Influence of rhythmic movement of the median nerve functionality in the upper limb in a patient with SCI: a case study [dissertation]. Torrelavega: Escuelas Universitarias Gimbernat; 2013.

17. Godoi J, Kerppers II, Rossi LP, et al. Electromyographic analysis of biceps brachii muscle following neural mobilization in patients with stroke. Electromyogr Clin Neurophysiol 2010;50:55-60.

18. Butler D, Jones M. Mobilisation of the nervous system. Edinburgh: Churchill Livingstone; 1991.

19. Marinzeck S. Neural mobilization: general aspects [Internet]. Campinas: Grupo Terapia Manual; 2010 [cited 2020 Mar 4]. Available from: https:// www.terapiamanual.com.br/site/noticias/arquivos/200912101725220.artigo_7.pdf.

20. Cowell IM, Phillips DR. Effectiveness of manipulative physiotherapy for the treatment of a neurogenic cervicobrachial pain syndrome: a single case study: experimental design. Man Ther 2002;7:31-8.

21. Villafane JH. Botulinum toxin type A combined with neurodynamic mobilization for lower limb spasticity: a case report. J Chiropr Med 2013;12:39-44.

22. Cha HK, Cho HS, Choi JD. Effects of the nerve mobilization technique on lower limb function in patients with poststroke hemiparesis. J Phys Ther Sci 2014;26:981-3. 